\title{
06 CONCORDANCE OF IDENTIFIED DRUGS IN INJURED DRIVERS USING LINKED MOTOR VEHICLE CRASH, EMERGENCY DEPARTMENT, AND INPATIENT HOSPITALISATION DATASETS
}

doi:10.1136/injuryprev-2012-040580d.6

T Bunn*, M Singleton, S Slavova, V Nicholson. Kentucky Injury Prevention and Research Center, University of Kentucky College of Public Health, Lexington, Kentucky, USA

Background Kentucky has the 6th highest drug overdose rate, 5th highest medical use rate of opioid pain relievers, and 11th highest opioid pain reliever sales rate in the USA (CDC, 2011).

Aims/Objectives/Purpose The present study was undertaken to identify the presence of drugs (illicit and prescription) and crash characteristics among drivers who were injured in a motor vehicle crash using linked emergency department (ED), inpatient hospitalisation (IH), and crash data.

Methods Kentucky CRASH data was probabilistically linked to emergency department datasets for years 2008-2009 and to inpatient hospitalisation datasets for years 2000-2009. Descriptive analyses were performed.

Results/Outcome Of the 47089 linked crash/ED-treated cases, there were: (1) 408 drivers diagnosed with non-dependent abuse of drugs (NDAD) in the ED; (2) 697 drivers cited for drugs in CRASH data (only 102 overlapped and were cited for drugs in both CRASH data and coded as NDAD in $\mathrm{IH}$ ); (3) 42 drivers diagnosed with multiple drugs; and (4) 98 drivers in very severe crashes. Of the 19466 total linked crash/IH cases, there were: (1) 1045 drivers diagnosed with NDAD in the IH; (2) 656 drivers cited for drugs in CRASH data (186 overlapped); (3) 250 drivers diagnosed with multiple drugs; and (4) 484 drivers in very severe crashes.

Significance/Contribution to the Field Community and workplace strategies focusing on comprehensive drug policies (eg, increased drug recognition training of police officers and increased traffic fines) and interventions (eg, improved prescription drug monitoring programmes, designated drivers) are necessary to reduce driving while under the influence of drugs. 